\title{
Subgame-Perfect $\epsilon$-Equilibria in Perfect Information Games with Common Preferences at the Limit
}

Citation for published version (APA):

Flesch, J., \& Predtetchinski, A. (2016). Subgame-Perfect $\epsilon$-Equilibria in Perfect Information Games with Common Preferences at the Limit. Mathematics of Operations Research, 41(4), 1208-1221. https://doi.org/10.1287/moor.2015.0774

Document status and date:

Published: 01/11/2016

DOI:

10.1287/moor.2015.0774

Document Version:

Publisher's PDF, also known as Version of record

Document license:

Taverne

Please check the document version of this publication:

- A submitted manuscript is the version of the article upon submission and before peer-review. There can be important differences between the submitted version and the official published version of record.

People interested in the research are advised to contact the author for the final version of the publication, or visit the DOI to the publisher's website.

- The final author version and the galley proof are versions of the publication after peer review.

- The final published version features the final layout of the paper including the volume, issue and page numbers.

Link to publication

\footnotetext{
General rights rights.

- You may freely distribute the URL identifying the publication in the public portal. please follow below link for the End User Agreement:

www.umlib.nl/taverne-license

Take down policy

If you believe that this document breaches copyright please contact us at:

repository@maastrichtuniversity.nl

providing details and we will investigate your claim.
}

Copyright and moral rights for the publications made accessible in the public portal are retained by the authors and/or other copyright owners and it is a condition of accessing publications that users recognise and abide by the legal requirements associated with these

- Users may download and print one copy of any publication from the public portal for the purpose of private study or research.

- You may not further distribute the material or use it for any profit-making activity or commercial gain

If the publication is distributed under the terms of Article $25 \mathrm{fa}$ of the Dutch Copyright Act, indicated by the "Taverne" license above, 


\title{
Subgame-Perfect $\epsilon$-Equilibria in Perfect Information Games with Common Preferences at the Limit
}

\author{
János Flesch \\ Department of Quantitative Economics, Maastricht University, 6200 MD Maastricht, the Netherlands, j.flesch@maastrichtuniversity.nl
}

Arkadi Predtetchinski

Department of Economics, Maastricht University, 6200 MD Maastricht, the Netherlands, a.predtetchinski@maastrichtuniversity.nl

\begin{abstract}
We prove the existence of a pure subgame-perfect epsilon-equilibrium, for every epsilon $>0$, in multiplayer perfect information games, provided that the payoff functions are bounded and exhibit common preferences at the limit. If, in addition, the payoff functions have finite range, then there exists a pure subgame-perfect 0 -equilibrium. These results extend and unify recent existence theorems for bounded and semicontinuous payoffs.
\end{abstract}

Keywords: perfect information games; subgame perfect equilibrium MSC2000 subject classification: Primary: 91A10, 91A18.

OR/MS subject classification: Primary: games/group decisions; secondary: noncooperative

History: Received July 11, 2014; revised June 29, 2015. Published online July 19, 2016.

1. Introduction. This paper extends and unifies two results on the existence of subgame perfect $\epsilon$-equilibrium in games of perfect information, by Flesch et al. [2] and Purves and Sudderth [15]. The setup is as follows: There are finitely many players. The game lasts infinitely many rounds, and at each round an active player chooses an action from an arbitrary non-empty set. This induces an infinite sequence of actions, called play. The payoff to each player is determined as a function of the play.

Flesch et al. [2] prove that a pure subgame perfect $\epsilon$-equilibrium exists for every $\epsilon>0$, provided that the payoff functions are bounded and lower semicontinuous. Purves and Sudderth [15] prove that a pure subgame perfect $\epsilon$-equilibrium exists for every $\epsilon>0$, provided that the payoff functions are bounded and upper semicontinuous. In both cases one takes the discrete topology on the set of actions and the product topology on the set of plays. The proofs of these two existence results use essentially different techniques.

We introduce a new geometric condition on the payoff functions, which we call common preferences at the limit, CPL for brevity. While we need CPL to state our existence result, it is instructive to consider a more restrictive version of the condition, called strong CPL. Roughly speaking, strong CPL says that, for every play $p$, if play $q$ is close enough to $p$, then up to a small error term, all players weakly prefer $p$ over $q$, or all players weakly prefer $q$ over $p$. In particular, strong CPL is satisfied if for every $p$ at least one of the following statements is true: (1) Every player's payoff function is lower semicontinuous at $p$, or (2) Every player's payoff function is upper semicontinuous at $p$. Consequently, strong CPL is satisfied if every player's payoff function is everywhere lower semicontinuous, or if every player's payoff function is everywhere upper semicontinuous. As a very special case, strong CPL holds whenever the payoff functions of the players are identical. This implies that there exist strong CPL payoff functions that are nowhere continuous, or that are not Borel measurable.

Our main condition, CPL is more permissive than strong CPL. Intuitively, CPL says that for every play $p$ the set of plays that are close to $p$ could be partitioned into two subsets in such a way that all plays in the subset containing $p$ are unanimously ranked below all plays in the other subset. Strong CPL corresponds to the case where one of the sets in the partition contains all plays weakly preferred to $p$, and the other set contains all plays worse than $p$.

Our main result is that a pure subgame perfect $\epsilon$-equilibrium exists for every $\epsilon>0$ if the payoff functions are bounded and satisfy CPL. If, in addition, the payoff functions have finite range, then there is a pure subgame perfect 0 -equilibrium.

The proof technique is as follows: We know that there exists a strategy profile that is immune to one-shot deviations (that is, deviations at a single time period), by a result by Flesch et al. [2]. The main challenge is ensuring that deviations at infinitely many time periods are not profitable.

The first step of the proof is to construct a strategy profile that is immune to one-shot deviations with an additional feature: Whenever in a given subgame there is a payoff vector that is unanimously preferred to any other feasible payoff vector, the strategy profile yields that payoff vector. The crucial step is then to show that if play $p$ is obtained by a deviation at infinitely many periods while $q$ is obtained by a deviation at finitely many periods, then $q$ is in the upper element of the partition relative to $p$, and therefore is unanimously weakly preferred to $p$. 
Related literature: Existence of a subgame perfect 0-equilibrium has been established under various conditions, see for example Fudenberg and Levine [7], Harris [8], and Maitra and Sudderth [12]. Typically, it is assumed that the payoff functions satisfy some form of continuity. Very general topological conditions for the existence of subgame perfect 0-equilibrium are given in Alós-Ferrer and Ritzberger [1].

For results on the existence of subgame perfect $\epsilon$-equilibrium, see Solan and Vieille [16], Solan [17], Flesch et al. [3], Laraki et al. [10], and Mashiah-Yaakovi [11], and Flesch and Predtetchinski [6, 5].

Solan and Vieille [16] present an example of a game that does not admit subgame perfect $\epsilon$-equilibrium in pure strategies for small $\epsilon$. Recently Flesch et al. [4] gave an example of a game that does not admit any subgame perfect $\epsilon$-equilibrium for small $\epsilon$, not even in behavior strategies. Both games have Borel measurable payoff functions, and only three different payoff vectors.

Structure of the paper: In Section 2, we define the model. In Section 3, we introduce the property of common preferences at the limit. In Section 4, we state and discuss the main results. Sections 5 and 6 present the formal proofs. Section 7 provides concluding remarks and discusses avenues for future research.

\section{The model.}

The game: Let $N=\{1, \ldots, n\}$ denote the set of players and let $A$ be an arbitrary non-empty set. Let $\mathbb{N}=\{0,1,2, \ldots\}$. We denote by $H$ the set of all finite sequences of elements of $A$, including the empty sequence $\emptyset$. We denote by $\mathscr{P}=A^{\mathbb{N}}$ the set of all infinite sequences of elements of $A$. The elements of $A$ are called actions, the elements of $H$ are called histories, and the elements of $\mathscr{P}$ are called plays. There is a function $\iota: H \rightarrow N$ that assigns an active player to each history. Furthermore, each player $i \in N$ is given a payoff function $u_{i}: \mathscr{P} \rightarrow \mathbb{R}$.

The game is played as follows: Player $\iota(\varnothing)$ chooses an action $a_{0}$. Suppose that up to period $t \in \mathbb{N}$ of the game the sequence $h=\left(a_{0}, \ldots, a_{t}\right)$ of actions has been chosen. Then player $\iota(h)$ chooses an action $a_{t+1}$. The chosen action is observed by all players. Continuing this way the players generate a play $p=\left(a_{0}, a_{1}, \ldots\right)$, and finally each player $i \in N$ receives payoff $u_{i}(p)$.

Strategies: We only consider pure strategies. Thus we omit the qualification "pure" in the sequel. A strategy for player $i$ is a function $\sigma_{i}: \iota^{-1}(i) \rightarrow A$, where $\iota^{-1}(i)$ is the set of histories where player $i$ moves. A strategy profile is a tuple $\left(\sigma_{1}, \ldots, \sigma_{n}\right)$ where each $\sigma_{i}$ is a strategy for player $i$. Given a strategy profile $\sigma=\left(\sigma_{1}, \ldots, \sigma_{n}\right)$ and a strategy $\eta_{i}$ for player $i$, we write $\sigma / \eta_{i}$ to denote the strategy profile obtained from $\sigma$ by replacing $\sigma_{i}$ with $\eta_{i}$.

For the concatenation of histories and actions we use the following notations: Take a history $h=\left(a_{0}, \ldots, a_{t}\right) \in$ $H$. For an action $b \in A$, we denote the sequence $\left(a_{0}, \ldots, a_{t}, b\right)$ by $(h, b)$ or $h b$. For a sequence of actions $\left(b_{0}, b_{1}, \ldots\right)$ we let $\left(h, b_{0}, b_{1}, \ldots\right)$ denote the play $\left(a_{0}, \ldots, a_{t}, b_{0}, b_{1}, \ldots\right)$.

We can identify a strategy profile $\sigma$ with a function $\sigma: H \rightarrow A$. Define the play induced by $\sigma$ starting from the history $h$, denoted by $\pi(\sigma, h)$, inductively as follows: Let $h_{0}=h$. If $h_{t}$ has been defined for some $t \geq 0$, then let $a_{t}=\sigma\left(h_{t}\right)$ and set $h_{t+1}=\left(h_{t}, a_{t}\right)$. Then $\pi(\sigma, h)=\left(h, a_{0}, a_{1}, \ldots\right)$. For the special case $h=\varnothing$, we write $\pi(\sigma)=\pi(\sigma, \varnothing)$.

Subgame perfect $\epsilon$-equilibrium: Let $\epsilon \geq 0$ be an error-term. A strategy profile $\sigma$ is called an $\epsilon$-equilibrium if no player can gain more than $\epsilon$ by a unilateral deviation, i.e., if for each player $i \in N$ and for each strategy $\sigma_{i}^{\prime}$ of player $i$ it holds that

$$
u_{i}(\pi(\sigma)) \geq u_{i}\left(\pi\left(\sigma / \sigma_{i}^{\prime}\right)\right)-\epsilon
$$

Subgame perfect $\epsilon$-equilibrium, $\epsilon$-SPE, requires that the strategy profile induces an $\epsilon$-equilibrium in every subgame. That is, for each history $h \in H$, each player $i \in N$, and each strategy $\sigma_{i}^{\prime}$ of player $i$ it holds that

$$
u_{i}(\pi(\sigma, h)) \geq u_{i}\left(\pi\left(\sigma / \sigma_{i}^{\prime}, h\right)\right)-\epsilon .
$$

A 0-equilibrium is called an equilibrium, and a 0-SPE is called an SPE. Without strong conditions, both can fail to exist, even in one-player games. Suppose for example that a player must choose a natural number, and his payoff is $1-1 / n$ if he chooses $n \in \mathbb{N}$. In this game, the player has obviously no optimal strategy, and therefore the game admits no equilibrium and no SPE. As a similar example, suppose that a player can choose to stop or to continue at time periods $t \in \mathbb{N}$; his payoff is $1-1 / t$ if he decides to stop the game at period $t$ and his payoff is 0 if he never stops. Note that for all $\epsilon>0$ both games admit an $\epsilon$-SPE, e.g., choosing any $n \geq 1 / \epsilon$ in the first game, and in the second game choosing to continue at periods $t<1 / \epsilon$ and choosing to stop at periods $t \geq 1 / \epsilon$. 
One-deviation immune profile: For our proof, the concept of a one-deviation immune profile is important. A strategy profile $\sigma$ is said to be one-deviation immune if

$$
u_{\iota(h)}(\pi(\sigma, h)) \geq u_{\iota(h)}(\pi(\sigma, h a)) \quad \text { for each } h \in H, \quad a \in A .
$$

Thus a strategy profile $\sigma$ is one-deviation immune if the active player cannot improve his payoff at any history $h$ by deviating from $\sigma$ only at history $h$. It follows by induction that a one-deviation immune profile automatically satisfies the finite-deviation property: No player can improve his payoff, in any subgame, by deviating from $\sigma$ at a finite number of histories. Any SPE is a one-deviation immune profile, but not vice versa.

The topological structure: We endow the action set $A$ with the discrete topology and the set of plays $\mathscr{P}$ with the product topology. The topology on $\mathscr{P}$ is completely metrizable, ${ }^{1}$ and a basis of this topology is formed by the cylinder sets $O(h)=\{p \in \mathscr{P}: h \prec p\}$ for $h \in H$, where for a history $h \in H$ and a play $p \in \mathscr{P}$ we write $h \prec p$ if $h$ is the initial segment of $p$. Thus a sequence of plays $\left(p_{n}\right)_{n \in \mathbb{N}}$ converges to a play $p$ precisely when for every $k \in \mathbb{N}$ there exists an $N_{k} \in \mathbb{N}$ such that $p_{n}$ coincides with $p$ on the first $k$ coordinates for every $n \geq N_{k}$.

A function $f: \mathscr{P} \rightarrow \mathbb{R}$ is said to be continuous at a play $p \in \mathscr{P}$ if, for every sequence of plays $\left(p_{n}\right)_{n \in \mathbb{N}}$ converging to $p$, we have $\lim _{n \rightarrow \infty} f\left(p_{n}\right)=f(p)$. Thus, $f$ is continuous at $p$ precisely when for every $\delta>0$ there is an $N_{\delta} \in \mathbb{N}$ such that if a play $q$ coincides with $p$ on the first $N_{\delta}$ coordinates then $|f(p)-f(q)| \leq \delta$. Intuitively, continuity of $f$ at $p$ says that changing the actions in $p$ at distant stages of the game has little effect on the value of $f$. Further, $f$ is said to be continuous if it is continuous at each play in $\mathscr{P}$.

A function $f: \mathscr{P} \rightarrow \mathbb{R}$ is said to be lower semicontinuous at a play $p \in \mathscr{P}$ if, for every sequence of plays $\left(p_{n}\right)_{n \in \mathbb{N}}$ converging to $p$, we have $\liminf _{n \rightarrow \infty} f\left(p_{n}\right) \geq f(p)$. We say that $f$ is lower semicontinuous if it is lower semicontinuous at each play in $\mathscr{P}$.

A function $f: \mathscr{P} \rightarrow \mathbb{R}$ is said to be upper semicontinuous at a play $p \in \mathscr{P}$ if, for every sequence of plays $\left(p_{n}\right)_{n \in \mathbb{N}}$ converging to $p$, we have $\lim _{\sup _{n \rightarrow \infty}} f\left(p_{n}\right) \leq f(p)$. We say that $f$ is upper semicontinuous if it is upper semicontinuous at each play in $\mathscr{P}$.

Existence results: Mertens and Neyman (cf. Mertens [14]) showed, by using the result of Martin [13], that if each player's payoff function is bounded and Borel measurable then an $\epsilon$-equilibrium exists for every $\epsilon>0$. An $\epsilon$-SPE (in pure strategies) does not necessarily exist under these conditions, as was demonstrated by examples in Solan and Vieille [16] and in Flesch et al. [4] (the latter has no $\epsilon-S P E$ even in behavior strategies). The following two sufficient conditions for the existence of $\epsilon-\mathrm{SPE}$ are important for this paper.

THeOREM 2.1 (Flesch ET AL. [2]). If for each player $i$ the payoff function $u_{i}$ is bounded and lower semicontinuous, then the game admits an $\epsilon-S P E$ for each $\epsilon>0$. If in addition, the payoff functions have finite range, then the game admits an SPE.

TheOrem 2.2 (Purves ANd SUdDerth [15]). If for each player $i$ the payoff function $u_{i}$ is bounded and upper semicontinuous, then the game admits an $\epsilon-S P E$ for each $\epsilon>0$. If in addition, the payoff functions have finite range, then the game admits an SPE.

Underlying Theorem 2.1 is the fact that any game with finitely many payoffs admits a one-deviation immune profile. $^{2}$

THEOREM 2.3 (FLESCH ET AL. [2]). If for each player $i$ the payoff function $u_{i}$ has finite range, then the game admits a one-deviation immune profile.

In general, a one-deviation immune profile need not be an SPE. However, if the payoff functions are lower semicontinuous, then a strategy profile is an SPE if and only if it is a one-deviation immune profile. Therefore Theorem 2.1 is an straightforward corollary of Theorem 2.3.

Orders on $\mathbb{R}^{n}:$ For vectors $v$ and $w$ in $\mathbb{R}^{n}$ we write $v \leq w$ if $\forall i \in N\left(v_{i} \leq w_{i}\right)$, we write $v<w$ if $v \leq w$ and $v \neq w$, and we write $v \ll w$ if $\forall i \in N\left(v_{i}<w_{i}\right)$.

\footnotetext{
${ }^{1}$ One can take for example the metric $d: \mathscr{P} \times \mathscr{P} \rightarrow \mathbb{R}$ which is defined for each $p, q \in \mathscr{P}$ as follows: If $p=q$ then $d(p, q)=0$. Otherwise, $d(p, q)=2^{-m(p, q)}$ where $m(p, q) \in \mathbb{N}$ is the first coordinate on which $p$ and $q$ differ.

${ }^{2}$ This statement is proven but not explicitly stated in Flesch et al. [2].
} 

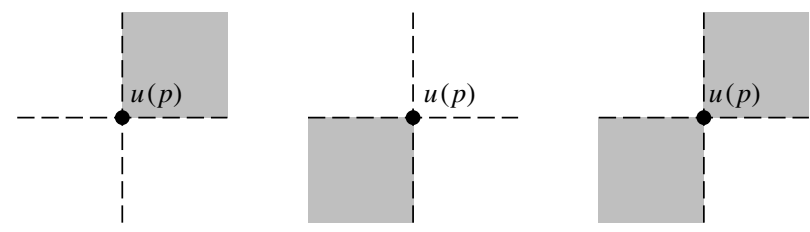

FIGURE 1. Lower semicontinuity, upper semicontinuity, and strong CPL.

3. Common preferences at the limit. In this section, we introduce a new geometric condition on the payoff functions, which we call common preferences at the limit. While we only need CPL to state our main results, a stronger version of the condition, called strong CPL, warrants some attention, as it encompasses a number of important special cases. Thus we begin our discussion with strong CPL, and then continue with CPL.

Consider a payoff function $u: \mathscr{P} \rightarrow \mathbb{R}^{n}$ given by $u(p)=\left(u_{1}(p), \ldots, u_{n}(p)\right)$. The graph of $u$ is the set $\{(p, u(p)) \mid p \in \mathscr{P}\}$, a subset of $\mathscr{P} \times \mathbb{R}^{n}$. Let $G_{u}$ denote the closure of the graph of $u$. Furthermore, for a play $p \in \mathscr{P}$ let $G_{u}(p)$ denote the $p$-section of $G_{u}$, that is $G_{u}(p)=\left\{v \in \mathbb{R}^{n} \mid(p, v) \in G_{u}\right\}$.

Flesch et al. [2] assume that each player $i$ 's payoff function $u_{i}$ is lower semicontinuous, or equivalently that for every $p \in \mathscr{P}$ and every $v \in G_{u}(p)$ it holds that $u(p) \leq v$. The assumption in Purves and Sudderth [15] is that each player $i$ 's payoff function $u_{i}$ is upper semicontinuous, or equivalently that for every $p \in \mathscr{P}$ and every $v \in G_{u}(p)$ it holds that $u(p) \geq v$. This brings us to the following definition:

Definition 3.1. The payoff function $u$ is said to exhibit strong common preferences at the limit, strong CPL, if for every $p \in \mathscr{P}$ and every $v \in G_{u}(p)$ it holds that $u(p) \leq v$ or $u(p) \geq v$.

The three panels of Figure 1 illustrate the definitions of lower semicontinuity, upper semicontinuity, and strong CPL, respectively, in the case of two players. In each case the set $G_{u}(p)$ should be contained in the shaded area.

Strong CPL is satisfied if for every play $p \in \mathscr{P}$ at least one of the following statements is true: (1) Every player $i$ 's payoff function $u_{i}$ is lower semicontinuous at $p$, or (2) Every player $i$ 's the payoff function $u_{i}$ is upper semicontinuous at $p$. Consequently, strong CPL is satisfied if every player's payoff function is everywhere lower semicontinuous, or if every player's payoff function is everywhere upper semicontinuous. Note however that strong CPL does not exclude that $u(p) \leq v$ for some $v \in G_{u}(p)$ while $u(p) \geq v$ for other $v \in G_{u}(p)$.

Here is an example of an infinite centipede game with payoff functions that exhibit strong CPL, but at some plays are neither lower semicontinuous nor upper semicontinuous.

Example 3.2. Consider the following 2-player game: The action set is $A=\{s, c\}$, where $s$ stands for stop and $c$ for continue. The players choose actions alternatingly, and as soon as a player chooses action $s$, the game ends. $^{3}$ The payoffs are $(-2,-1)$ if player 1 ends the game, $(1,2)$ if player 2 ends the game, and $(0,0)$ if the players never play action $s$. This is shown in the following figure:

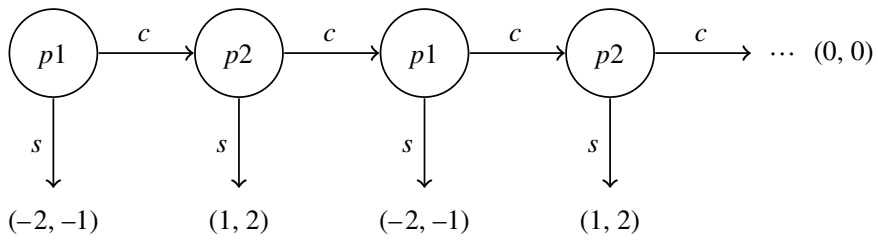

The payoff functions are neither lower nor upper semicontinuous at the play $p=(c, c, \ldots)$, and are continuous everywhere else. To verify strong CPL, it suffices to consider the play $p$. The set $G_{u}(p)$ consists of the vectors $(0,0),(-2,-1)$, and $(1,2)$, but since $u(p)=(0,0) \leq(1,2)$ and $u(p)=(0,0) \geq(-2,-1)$, strong CPL holds. $\triangleleft$

As shown by the example above, strong CPL payoff functions need not be semicontinuous. In fact, strong CPL payoff functions might be very "irregular." There exist strong CPL payoff functions that are nowhere continuous, and there exist strong CPL payoff functions that are not Borel measurable (with respect to the chosen topology on $\mathscr{P})$. Indeed, let $f: \mathscr{P} \rightarrow \mathbb{R}$ be an arbitrary function. Define $u_{1}=\cdots=u_{n}=f$. In this case, all payoff functions are identical, so strong CPL is trivially satisfied.

We now turn to our main definition. 
Definition 3.3. The payoff function $u$ is said to exhibit common limit preferences, CPL, if there exists a function $s: \mathscr{P} \rightarrow \mathbb{R}^{n}$ such that for every $p \in \mathscr{P}$ the following are satisfied: $(1) u(p) \leq s(p)$; $(2)$ There is $v \in G_{u}(p)$ such that $s(p) \leq v$; and (3) For every $v \in G_{u}(p)$ either $v \leq s(p)$ or $s(p) \leq v$. In this case, the function $s$ is called a splitting function associated with $u$.

Note that $u$ exhibits strong CPL exactly when it exhibits CPL with the splitting function $s=u$. The following game exhibits CPL, but not strong CPL:

ExAmple 3.4. Consider the following 2-player game: The action set is $A=\{s, c\}$, where $s$ stands for stop and $c$ for continue. The players choose actions alternatingly, and as soon as a player chooses action $s$, the game ends. The payoffs are $(1,1)$ if the game ends at a period $4 k,(-1,1)$ if it ends at a period $4 k+1,(1,-1)$ if it ends at a period $4 k+2,(2,2)$ if it ends at a period $4 k+3$, and $(0,0)$ if the game never ends. This is shown in the following figure:

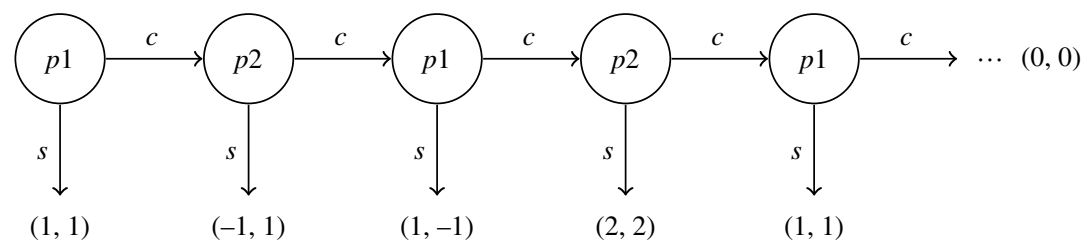

Again, to verify (strong) CPL, it suffices to consider the play $p=(c, c, \ldots)$. We have $v \in G_{u}(p)$ for any of the five payoff vectors $v$ in the game. Since $u(p)=(0,0)$ and $(1,-1)$ are not $\leq-$ comparable, the payoffs do not satisfy strong CPL. However, by choosing $s(p)=(1,1)$, we find that the payoffs do exhibit CPL. See Figure 2. $\triangleleft$

Examples of games that do not have an $\epsilon-\mathrm{SPE}$ in pure strategies are given in Solan and Vieille [16] and Flesch et al. [4]. In both examples the payoffs violate CPL, as shown below.

Example 3.5 (Solan and Vieille [16]). Consider the following 2-player game: The action set is $A=$ $\{s, c\}$, where $s$ stands for stop and $c$ for continue. The players choose actions in turn, and as soon as a player chooses action $s$, the game ends. The payoffs are $(-1,2)$ if player 1 ends the game, $(-2,1)$ if player 2 ends the game, and $(0,0)$ if the players never play action $s$. This is shown in the following figure:

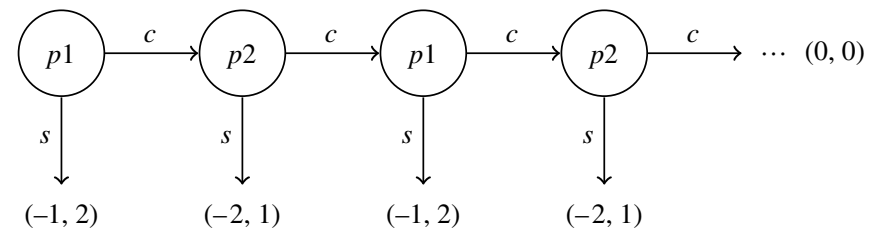

Since for the play $p=(c, c, \ldots)$ the set $G_{u}(p)$ consists of the points $(-2,1),(-1,2)$, and $(0,0)$, the payoff function $u$ cannot satisfy conditions (1)-(3) of CPL for any splitting function $s$. Note that, as Solan and Vieille argue, this game admits no pure $\epsilon-\mathrm{SPE}$, but it does have an $\epsilon-\mathrm{SPE}$ in behavior strategies. $\triangleleft$

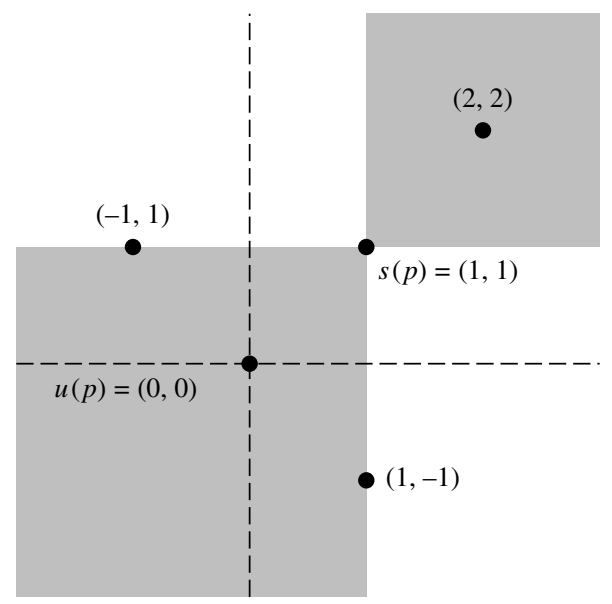

FIgURE 2. The game in Example 3.4. 
Example 3.6 (Flesch et Al. [4]). Consider the following 2-player game: The action set is $A=\{1,2\}$. Player 1 starts the game. The active player decides who the next active player is by choosing the corresponding action. The payoffs are $(-1,2)$ if player 2 is active only finitely many times, $(-2,1)$ if player 1 is active only finitely many times, and $(0,0)$ if both players are active infinitely many times.

For any play $p$ the set $G_{u}(p)$ consists of the points $(-2,1),(-1,2)$, and $(0,0)$, so the payoff function $u$ cannot satisfy conditions (1)-(3) of CPL for any splitting function $s$. As Flesch et al. argue, this game admits no $\epsilon-\mathrm{SPE}$ for small $\epsilon$, not even in behavior strategies. $\triangleleft$

Suppose that the payoff function $u$ exhibits CPL, and let $s$ be an associated splitting function. Consider a play $p$ such that for each player $i \in N$, player $i$ 's payoff function $u_{i}$ is upper semicontinuous at $p$. Then $v \leq u(p)$ for each $v \in G_{u}(p)$. Hence conditions (1) and (2) of Definition 3.3 imply that $u(p)=s(p)$. We discuss the connection between upper semicontinuity and splitting functions in more detail in $\$ 7$.

A zero-sum game satisfies CPL if and only if the payoff function is continuous. This could be considered a limitation of CPL as every zero-sum game has an $\epsilon$-SPE provided only that the payoff function is bounded and Borel measurable, a result that could be proven using Martin's Borel determinacy (1975).

4. The main results. In this section, we present our main results.

THEOREM 4.1. If the payoff function $u=\left(u_{1}, \ldots, u_{n}\right)$ is bounded and exhibits common preferences at the limit, then the game admits an $\epsilon-S P E$ for every $\epsilon>0$.

Note that Theorem 4.1 does not claim that there exists an SPE (see examples in §2). We do obtain existence of SPE if the payoff function has finite range in addition.

THEOREM 4.2. If the payoff function $u=\left(u_{1}, \ldots, u_{n}\right)$ has finite range and exhibits common preferences at the limit, then the game admits an SPE.

The proof of Theorem 4.2 uses a result in Flesch et al. [2], cf. Theorem 2.3 above on the existence of a onedeviation immune profile. As already mentioned, the one-deviation property automatically implies, by induction, the finite-deviation property, in the sense that no player $i$ can improve his payoff, in any subgame, by deviating from $\sigma_{i}$ finitely many times. The main challenge is to make sure that deviating infinitely many times is not profitable either.

The proof of Theorem 4.2 proceeds in a sequence of steps. First, we argue that we can perturb the payoffs slightly to obtain a new payoff function without ties (Lemma 5.1). Second, we construct a one-deviation immune profile $\sigma$ with an additional feature: In a subgame, whenever there is a feasible payoff, say $v$, that is unanimously preferred to any other feasible payoff, $\sigma$ leads to the payoff of $v$ (Lemma 5.2, this is where we use Theorem 2.3). Finally, we argue that $\sigma$ is an SPE. The crucial point is that for any play $p$ and any sufficiently long history $h \prec p$, the payoff induced by $\sigma$ starting at $h$ is bounded below by $s(p)$, and therefore also by $u(p)$. This implies that a deviation leading to $p$ cannot be profitable.

Subsequently we show that each bounded CPL payoff function $u$ admits a CPL discretization, that is, a CPL payoff function $\bar{u}$ with finite range that is $\epsilon$-close to $u$. Theorem 4.1 then follows at once from Theorem 4.2.

Throughout the paper we assume the Axiom of Choice.

5. The proof of Theorem 4.2. The payoff function $u$ is said to have a tie if there are two payoffs $v$ and $w$ in the range of $u$ such that $v \neq w$ but $v(i)=w(i)$ for some player $i \in\{1, \ldots, n\}$.

Lemma 5.1. Suppose that the payoff function u has a finite range and exhibits CPL. Then, there is a payoff function $u^{\prime}$ such that (1) $u^{\prime}$ has a finite range without ties, (2) $u^{\prime}$ exhibits CPL, and (3) any SPE with respect to $u^{\prime}$ is also an SPE with respect to $u$.

Proof. Let $u$ have a finite range and exhibit CPL. Clearly, we can assume without loss of generality that the splitting function $s$ has a finite range, too. Let $v_{1}, \ldots, v_{m}$ be an enumeration of the union of the range of $u$ and the range of $s$ such that if $v_{j} \leq v_{k}$ then $j \leq k$. Let $\delta>0$ be so small that whenever $v_{j}(i) \neq v_{k}(i)$, for some $j, k \in\{1, \ldots, m\}$ and $i \in\{1, \ldots, n\}$, then $(1 / m) \cdot\left|v_{j}(i)-v_{k}(i)\right|>\delta$.

By construction, for all $j, k \in\{1, \ldots, m\}$ and all $i \in\{1, \ldots, n\}$ we have the following two properties: (A) If $v_{j}(i)<v_{k}(i)$, then $v_{j}(i)+j \delta<v_{k}(i)<v_{k}(i)+k \delta$, and (B) If $v_{j}(i)=v_{k}(i)$ and $j<k$ then $v_{j}(i)+j \delta<v_{k}(i)+k \delta$.

Define new functions $u^{\prime}$ and $s^{\prime}$ as follows: For each $p \in \mathscr{P}$ let $u^{\prime}(p)=v_{k}+k \delta^{n}$ if $u(p)=v_{k}$. Similarly, let $s^{\prime}(p)=v_{k}+k \delta^{n}$ if $s(p)=v_{k}$, where $\delta^{n}=(\delta, \ldots, \delta) \in \mathbb{R}^{n}$. The union of the range of $u^{\prime}$ and the range of $s^{\prime}$ is the set of $m$ payoff vectors $v_{1}+\delta^{n}, \ldots, v_{m}+m \delta^{n}$. 
To see that $u^{\prime}$ has no ties suppose that $v_{j}(i)+j \delta=v_{k}(i)+k \delta$ for some player $i$. However, then by (A) and (B) it holds that $v_{j}(i)=v_{k}(i)$ and $j=k$, so $v_{j}+j \delta^{n}=v_{k}+k \delta^{n}$. Thus $u^{\prime}$ satisfies property (1) of the lemma.

To see that $u^{\prime}$ has property (2), note that $v_{j} \leq v_{k}$ implies that $j \leq k$, and consequently that $v_{j}+j \delta^{n} \leq v_{k}+k \delta^{n}$. Hence for $p, q \in \mathscr{P}$ if $u(p) \leq s(q)$ then $u^{\prime}(p) \leq s^{\prime}(q)$, and if $u(p) \geq s(q)$ then $u^{\prime}(p) \geq s^{\prime}(q)$. Thus $u^{\prime}$ exhibits CPL with $s^{\prime}$ as an associated splitting function. Property (2) follows.

Finally, to show that property (3) holds, observe that by (A) if $u_{i}(p)<u_{i}(q)$ for some plays $p, q \in \mathscr{P}$, then also $u_{i}^{\prime}(p)<u_{i}^{\prime}(q)$.

Given a history $h \in H$ let $U(h)=\{u(q) \mid q \succ h\}$. For histories $h$ and $h^{\prime}$ we write $h \preceq h^{\prime}$ if $h^{\prime}=h$ or $h^{\prime}$ extends $h$. In this case $U(h) \supseteq U\left(h^{\prime}\right)$.

The next lemma says that there is a one-deviation immune profile $\sigma$ such that whenever in a subgame starting at $h$ there is a feasible payoff, say $v^{h}$, that is unanimously preferred to any other feasible payoff, then $\sigma$ leads to the payoff of $v^{h}$ in the given subgame. The lemma applies to any payoff function with finitely many values, and does not use CPL.

Lemma 5.2. Suppose that the payoff function $u$ has finite range. Then the game admits a one-deviation immune profile $\sigma$ with the following property: If for a history $h \in H$ there exists a payoff $v^{h} \in U(h)$ such that $v \leq v^{h}$ for every $v \in U(h)$, then $u(\pi(\sigma, h))=v^{h}$.

Proof. Let $M$ denote the set of histories $h \in H$ for which there exists a payoff $v^{h} \in U(h)$ such that $v^{\prime} \leq v^{h}$ for every $v^{\prime} \in U(h)$. Clearly, there exists a strategy profile $\tau$ such that $u(\pi(\tau, h))=v^{h}$ for every $h \in M$.

Let $M^{+}$denote the set of histories of the form $\left(h^{\prime}, a\right)$ where $h^{\prime} \in M$ but $\left(h^{\prime}, a\right) \notin M$. As a special case, we also include the root history $\emptyset$ in $M^{+}$if $\emptyset \notin M$. For every history $h \notin M$, let $\phi(h)$ denote the longest history $h^{\prime}$ with $h^{\prime} \preceq h$ and $h^{\prime} \in M^{+}$.

For every $h \in M^{+}$, consider the payoff function $w^{h}$ which we define for every play $p \in \mathscr{P}$ as follows:

Case 1: If $p$ begins with $h$ and enters $M$ afterwards, i.e., if $h \prec p$ and there is a history $h^{\prime} \in M$ such that $h \prec h^{\prime} \prec p$. In this case, assume that $h^{\prime}$ is minimal with this property. We set $w^{h}(p)=v^{h^{\prime}}$.

Case 2: Otherwise, we set $w^{h}(p)=u(p)$.

By Theorem 2.3, there is a one-deviation immune profile $\tau^{h}$ for the payoff function $w^{h}$.

Now we are ready to define the strategy profile $\sigma$. Intuitively, whenever play is in $M$ then $\sigma$ follows $\tau$ and gives the unanimously preferred payoff; whenever play leaves $M$, say at a history $h^{\prime} \in M^{+}$, then $\sigma$ follows $\tau^{h^{\prime}}$ as long as $M$ is not entered again. Now we provide a formal definition. Take a history $h \in H$. We distinguish two cases:

Case 1: If $h \in M$. In this case, we set $\sigma(h)=\tau(h)$.

Case 2: If $h \notin M$. In this case, we set $\sigma(h)=\tau^{\phi(h)}(h)$.

Since $\sigma$ coincides with $\tau$ on $M$, we have $u(\pi(\sigma, h))=u(\pi(\tau, h))=v^{h}$ for all $h \in M$, so it is clear that $\sigma$ satisfies that additional property in the lemma. It remains to verify that $\sigma$ is a one-deviation immune profile. Clearly, no single deviation at a history $h \in M$ can be profitable, since $u(\pi(\sigma, h))=v^{h}$. Now suppose that $h \notin M$. Note that

$$
u(\pi(\sigma, h))=w^{\phi(h)}\left(\pi\left(\tau^{\phi(h)}, h\right)\right) .
$$

Indeed, if $\pi(\sigma, h)$ never enters $M$ after $h$ then $\pi(\sigma, h)=\pi\left(\tau^{\phi(h)}, h\right)$ and hence by case 2 of the definition of $w^{h},(2)$ holds. Otherwise $\pi(\sigma, h)$ and $\pi\left(\tau^{\phi(h)}, h\right)$ enter $M$ at the very same history $h^{\prime \prime} \in M$ and then both $u(\pi(\sigma, h))$ and $w^{\phi(h)}\left(\pi\left(\tau^{\phi(h)}, h\right)\right)$ are equal to $v^{h^{\prime \prime}}$, and (2) holds again. For every history $h \notin M$ and action $a \in A$ we have

$$
u(\pi(\sigma, h a))=w^{\phi(h)}\left(\pi\left(\tau^{\phi(h)}, h a\right)\right) .
$$

Indeed, if $h a \notin M$, this is an instance of (2), and if $h a \in M$, then the left and the right-hand sides are equal to $v^{h a}$. Because $\tau^{\phi(h)}$ is a one-deviation immune profile for $w^{\phi(h)}$, it follows from (2) and (3) that

$$
u_{\iota(h)}(\pi(\sigma, h))=w_{\iota(h)}^{\phi(h)}\left(\pi\left(\tau^{\phi(h)}, h\right)\right) \geq w_{\iota(h)}^{\phi(h)}\left(\pi\left(\tau^{\phi(h)}, h a\right)\right)=u_{\iota(h)}(\pi(\sigma, h a)) .
$$

Hence, $\sigma$ is a one-deviation immune profile.

Example 5.3. Consider the following 1-player game. The action set is $A=\{s, c\}$, where $s$ stands for stop and $c$ for continue. As soon as the player chooses action $s$ the game ends with payoff 0 , whereas always playing action $c$ gives payoff 1 . The strategy that always chooses action $s$ is clearly a one-deviation immune profile, even though the optimal payoff is 1 . Note that always choosing action $c$ is also a one-deviation immune profile, yielding this optimal payoff, and that this strategy now also satisfies the additional requirement of Lemma 5.2. 
Suppose that the payoff function $u$ has finite range. For a play $p$ let $h_{k}$ be the prefix of $p$ of length $k$. Clearly $U\left(h_{0}\right) \supseteq U\left(h_{1}\right) \supseteq \cdots$ is a non-increasing sequence of finite sets. Hence there is a $k \in \mathbb{N}$ such that $U\left(h_{k}\right)=U\left(h_{k+1}\right)=\cdots$. Clearly, $G_{u}(p)=U\left(h_{k}\right)$. Letting $k$ be the least number with this property, we set $h(p)=h_{k}$. Let $H(p)=\{h \in H \mid h(p) \preceq h \prec p\}$.

Define $X$ as the set consisting of all plays $p \in \mathscr{P}$ such that $u(p) \geq v$ for each $v \in G_{u}(p)$. We remark that $p \in X$ if and only if for every player $i$ the payoff function $u_{i}$ is upper semicontinuous at $p$. Note also that if $\sigma$ is a strategy profile as in Lemma 5.2 then

$$
u(\pi(\sigma, h))=u(p) \text { for every } p \in X \text { and } h \in H(p) .
$$

Lemma 5.4. Suppose that the payoff function $u$ has finite range and exhibits CPL. Let $s$ be a splitting function for $u$. Then, for each $p \in \mathscr{P}$ and $h \in H(p)$, there is $a q \succ h$ such that $q \in X$ and $s(p) \leq u(q)$.

Proof. Take a $p \in \mathscr{P}$ and an $h \in H(p)$. Take a $y \in G_{u}(p)$ such that $\neg \exists v \in G_{u}(p)(y<v)$, i.e., some $y$ which is maximal in $G_{u}(p)$ with respect to $\leq$. Such a vector $y$ exists since $G_{u}(p)$ is finite. First we argue that $s(p) \leq y$. By CPL either $y<s(p)$ or $s(p) \leq y$. Suppose $y<s(p)$. Take a $v \in G_{u}(p)$ such that $s(p) \leq v$. However, then $y<v$, contradicting the choice of $y$. Hence $s(p) \leq y$, as desired.

By the choice of $h$ we have $G_{u}(p)=U(h)$. Hence $y \in U(h)$, so there is a $q \succ h$ such that $y=u(q)$. Note that $G_{u}(q) \subseteq U(h)=G_{u}(p)$.

We know that $s(p) \leq y=u(q) \leq s(q)$. Take a $v \in G_{u}(q)$ such that $s(q) \leq v$. Since it is not the case that $y<v$, we must have $y=v$, and we conclude that $y=s(q)$. Now for each $w \in G_{u}(q)$ either $w \leq s(q)$ or $s(q)<w$. The latter is impossible as it implies $y<w$. We thus conclude that $w \leq s(q)=y=u(q)$. This proves that $q \in X$.

Lemma 5.5. Suppose that the payoff function $u$ has finite range without ties, and exhibits CPL. Let $s$ be a splitting function for $u$. Let the strategy profile $\sigma$ be as in Lemma 5.2. Then $u(\pi(\sigma, h)) \geq s(p)$ for each $p \in \mathscr{P}$ and each $h \in H(p)$.

Proof. Take a $p \in \mathscr{P}$ and an $h \in H(p)$. By Lemma 5.4 there is a $q \succ h$ such that $q \in X$ and $s(p) \leq u(q)$.

If $h(q) \preceq h$ then $h \in H(q)$ and hence $u(q)=u(\pi(\sigma, h))$ by (4). Therefore $s(p) \leq u(\pi(\sigma, h))$, as desired.

Suppose now that $h \prec h(q)$. Write $h(q)=h a_{1} \ldots a_{K}$ and let $h_{0}=h$, and for each $k \in\{1, \ldots, K\}$ let $h_{k}=h_{k-1} a_{k}$. In particular $h_{K}=h(q)$. We show that

$$
u\left(\pi\left(\sigma, h_{k}\right)\right) \geq s(p)
$$

for all $k \in\{0, \ldots, K\}$. For $k=K$, inequality (5) holds because $s(p) \leq u(q)=u(\pi(\sigma, h(q)))$, where the equality is a consequence of (4). Suppose (5) holds for some $k \geq 1$. We prove that (5) also holds for $k-1$. Letting $i=\iota\left(h_{k-1}\right)$ we have the chain of inequalities

$$
u_{i}\left(\pi\left(\sigma, h_{k-1}\right)\right) \geq u_{i}\left(\pi\left(\sigma, h_{k-1} a_{k}\right)\right)=u_{i}\left(\pi\left(\sigma, h_{k}\right)\right) \geq s_{i}(p),
$$

where the first inequality is an instance of (1), and the second inequality follows from the induction hypothesis. Suppose first that $u_{i}\left(\pi\left(\sigma, h_{k-1}\right)\right)>s_{i}(p)$. Then since $h_{k-1} \in H(p)$, CPL implies that $u\left(\pi\left(\sigma, h_{k-1}\right)\right) \geq s(p)$. Suppose now that $u_{i}\left(\pi\left(\sigma, h_{k-1}\right)\right)=s_{i}(p)$. Then by (6), $u_{i}\left(\pi\left(\sigma, h_{k-1}\right)\right)=u_{i}\left(\pi\left(\sigma, h_{k}\right)\right)$, and since $u$ has no ties, $u\left(\pi\left(\sigma, h_{k-1}\right)\right)=u\left(\pi\left(\sigma, h_{k}\right)\right)$. Hence the induction hypothesis implies that $u\left(\pi\left(\sigma, h_{k-1}\right)\right) \geq s(p)$, as desired.

Proof of Theorem 4.2. Let $u$ be a payoff function with finite range and CPL with a splitting function $s$. By Lemma 5.1 we can assume that $u$ has no ties. Let the strategy profile $\sigma$ be as in Lemma 5.2. We show that $\sigma$ is an SPE.

Let $h$ be any history and $\eta_{i}$ a strategy for player $i$. We want to prove that

$$
u_{i}(\pi(\sigma, h)) \geq u_{i}\left(\pi\left(\sigma / \eta_{i}, h\right)\right) .
$$

Write $\eta=\sigma / \eta_{i}$. Let $p=\pi(\eta, h)$ and let $h_{0}, h_{1}, h_{2}, \ldots$ be the successive enumeration of the initial segments of the play $p$ starting from $h$, that is $h_{0}=h$ and $h_{k+1}=h_{k} \eta\left(h_{k}\right)$ for each $k$. We have

$$
u_{i}\left(\pi\left(\sigma, h_{k}\right)\right) \geq u_{i}\left(\pi\left(\sigma, h_{k} \eta\left(h_{k}\right)\right)\right)=u_{i}\left(\pi\left(\sigma, h_{k+1}\right)\right)
$$

Indeed, at histories $h_{k}$ that belong to player $i$, the inequality is an instance of (1) with $a=\eta\left(h_{k}\right)$. At histories $h_{k}$ that do not belong to player $i$, the inequality holds as we have $\eta\left(h_{k}\right)=\sigma\left(h_{k}\right)$ and so $\pi\left(\sigma, h_{k}\right)=\pi\left(\sigma, h_{k} \eta\left(h_{k}\right)\right)$.

Hence we have a non-increasing chain of payoffs:

$$
u_{i}\left(\pi\left(\sigma, h_{0}\right)\right) \geq u_{i}\left(\pi\left(\sigma, h_{1}\right)\right) \geq \cdots \geq u_{i}\left(\pi\left(\sigma, h_{k}\right)\right) \geq \cdots
$$

Moreover for large $k$ we have $h_{k} \in H(p)$ and hence by Lemma 5.5, $u_{i}\left(\pi\left(\sigma, h_{k}\right)\right) \geq s_{i}(p) \geq u_{i}(p)$. Thus $u_{i}\left(\pi\left(\sigma, h_{0}\right)\right) \geq u_{i}(p)$, as desired. 


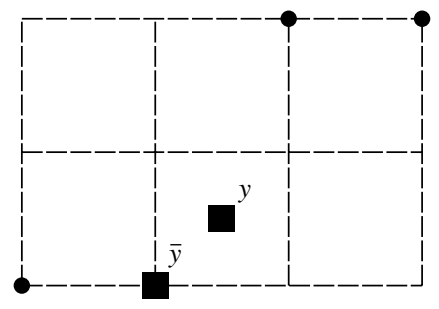

FIGURE 3. Illustration of Lemma 6.3.

6. The proof of Theorem 4.1. For a vector $v$ in $\mathbb{R}^{n}$ let $\|v\|=\max \left\{\left|v_{1}\right|, \ldots,\left|v_{n}\right|\right\}$. Let $\mathscr{G}$ be the set of functions $g: \mathscr{P} \rightarrow \mathbb{R}^{n}$. A function $\bar{g} \in \mathscr{G}$ is said to be an $\epsilon$-discretization of the payoff function $g \in \mathscr{G}$ if $\bar{g}$ has finite range and $\|g(p)-\bar{g}(p)\|<\epsilon$ for each $p \in \mathscr{P}$. We say that a subset $\mathscr{G}^{\prime}$ of $\mathscr{G}$ is discretizable if for each $g \in \mathscr{G}^{\prime}$ and each $\epsilon>0, g$ has an $\epsilon$-discretization $\bar{g}$ that is also an element of $\mathscr{G}^{\prime}$.

In this section we prove the following two related results:

THEOREM 6.1. The set of bounded strong CPL functions is discretizable.

THEOREM 6.2. The set of bounded CPL functions is discretizable.

Once Theorem 6.2 is proven, Theorem 4.1 follows from Theorem 4.2. Indeed, if $\bar{u}$ is an $\epsilon$-discretization of $u$, then any SPE relative to $\bar{u}$ is a $2 \epsilon-\mathrm{SPE}$ relative to $u$.

Flesch et al. [2] prove that the set of bounded and lower semicontinuous payoff functions is discretizable, while Purves and Sudderth [15] show that the set of bounded and upper semicontinuous payoff functions is discretizable. Both proofs are straightforward. For example if $u$ is bounded and lower semicontinuous, then a lower semicontinuous $\epsilon$-discretization of $u$ is given by $\bar{u}_{i}(p)=\epsilon\left\lfloor u_{i}(p) / \epsilon\right\rfloor$, where $\lfloor a\rfloor$ is the largest integer strictly smaller than $a$.

Unlike the case of semicontinuous functions, our proof is more involved. In particular, we were unable to construct an $\epsilon$-discretization of $u$ of the form $\bar{u}(p)=t(u(p))$ for any function $t: \mathbb{R}^{n} \rightarrow \mathbb{R}^{n}$. In our construction $\bar{u}(p)$ generally depends not only on $u(p)$ but also on $p$ itself.

Given a partial order $E$ on a set $X$ we say that the points $x$ and $y$ of $X$ are $E$-comparable if $x E y$ or $y E x$. A subset $S$ of $X$ is said to be an $E$-chain if for all $x, y \in S$ the points $x$ and $y$ are $E$-comparable. For the points $v$ and $y$ of $\mathbb{R}^{n}$ we write $v \ll_{\epsilon} y$ if $\forall i \in N\left(v_{i}<y_{i}+\epsilon\right)$ and we write $v \leq_{\epsilon} y$ if $\forall i \in N\left(v_{i} \leq y_{i}+\epsilon\right)$.

Fix a bounded function $g \in \mathscr{G}$ with strong CPL, and a bounded function $u \in \mathscr{G}$ with CPL. Fix also an $\epsilon>0$. We shall construct a strong CPL $\epsilon$-discretization of $g$ and a CPL $\epsilon$-discretization of $u$.

Let $M$ be a positive multiple of $\epsilon$ such that $-M \leq g_{i}(p) \leq M$ and $-M \leq u_{i}(p) \leq M$ for all $p \in \mathscr{P}$ and all $i$. Let $F_{\epsilon}$ be the set of points $x \in[-M, M]^{n}$ such that each coordinate $x_{i}$ is a multiple of $\epsilon$. The set $F_{\epsilon}$ serves as a grid, and the range of the discretized payoff functions will be contained in it.

Lemma 6.3. Let $C \subseteq F_{\epsilon}$ be $a \leq-$ chain and $y \in[-M, M]^{n}$. Suppose that for each $x \in C$, the points $x$ and $y$ are $\ll_{\epsilon}$-comparable. Then there exists an element $\bar{y} \in F_{\epsilon}$ such that

- $\|\bar{y}-y\|<\epsilon$ and

- for each $x \in C$, if $x \ll_{\epsilon} y$ then $x \leq \bar{y}$ and if $\neg\left(x \ll_{\epsilon} y\right)$ then $\bar{y} \leq x$.

Figure 3 illustrates the idea behind Lemma 6.3 for the two-player case. Points of the grid are elements of $F_{\epsilon}$. The black dots are the elements of $C$. Note that $C$ is indeed an $\leq-$ chain. Also pictured is a point $y \in[-M, M]^{2}$, and a point $\bar{y} \in F$ satisfying the condition of the lemma. The point $\bar{y}$ is also the point chosen under the construction in the proof of the lemma below.

Proof of Lemma 6.3. We assume that there is an $x \in C$ such that $x \ll_{\epsilon} y$. Otherwise add the point $(-M, \ldots,-M)$ to the set $C$. Let $x(1), \ldots, x(k)$ be an enumeration of $C$ such that $x(1) \leq \cdots \leq x(k)$. Let $m$ be the maximal number such that $x(m) \ll_{\epsilon} y$.

Let $a_{i}$ be the largest multiple of $\epsilon$ that is smaller than or equal to $y_{i}$, and $b_{i}$ be the smallest multiple of $\epsilon$ that is greater than or equal to $y_{i}$. Then $a_{i} \leq y_{i} \leq b_{i}$ and $b_{i}-a_{i}$ is 0 or $\epsilon$. Define

$$
\bar{y}_{i}= \begin{cases}a_{i} & \text { if } x_{i}(m) \leq y_{i} \\ b_{i} & \text { otherwise }\end{cases}
$$

Note that $\bar{y}$ is in $F_{\epsilon}$. 
We show that $x(\ell) \leq \bar{y}$ if $\ell \leq m$ and $\bar{y} \leq x(\ell)$ if $m<\ell$.

Consider an element $x(\ell)$ of $C$ with $\ell \leq m$. We have $x(\ell) \leq x(m)$ and we show that $x(m) \leq \bar{y}$. Take an $i$. Suppose first $x_{i}(m) \leq y_{i}$. Then $\bar{y}_{i}=a_{i}$. Since $x_{i}(m)$ is a multiple of $\epsilon$ we have $x_{i}(m) \leq a_{i}$ by the definition of $a_{i}$. Hence $x_{i}(m) \leq \bar{y}_{i}$. Now suppose that $y_{i}<x_{i}(m)$ so that $\bar{y}_{i}=b_{i}$. Then $y_{i}<x_{i}(m)<y_{i}+\epsilon$. Therefore $b_{i}=x_{i}(m)$ and hence $\bar{y}_{i}=x_{i}(m)$.

Consider an element $x(\ell)$ of $C$ with $m<\ell$. Since it is not the case that $x(\ell) \ll_{\epsilon} y$ we have $y \ll_{\epsilon} x(\ell)$. We argue that $\bar{y} \leq x(\ell)$. Take any $i$. Suppose first that $x_{i}(m) \leq y_{i}$, in which case $\bar{y}_{i}=a_{i}$. Then $a_{i} \leq y_{i}<x_{i}(\ell)+\epsilon$, and hence $a_{i}<x_{i}(\ell)+\epsilon$, therefore $a_{i} \leq x_{i}(\ell)$. Hence $\bar{y}_{i} \leq x_{i}(\ell)$. Now suppose that $y_{i}<x_{i}(m)$, in which case $\bar{y}_{i}=b_{i}$. Then $y_{i}<x_{i}(m) \leq x_{i}(\ell)$. Since $x_{i}(\ell)$ is a multiple of $\epsilon$, the definition of $b_{i}$ implies that $b_{i} \leq x_{i}(\ell)$. Thus $\bar{y}_{i} \leq x_{i}(\ell)$.

Lemma 6.4 below roughly speaking states the following: Take a play $q$. If $p$ is close to $q$ and if vector $x$ is close to the payoff vector $g(q)$, then $g(p)$ and $x$ are $\ll_{\epsilon}$-comparable. This follows since by strong CPL, if $p$ is close to $q$, then $g(p)$ is "almost" $\leq-$ comparable to $g(q)$.

Lemma 6.4. For each $q \in \mathscr{P}$, there exists a history $\ell(q) \prec q$ such that, for each $p \succ \ell(q)$ and each $x \in F_{\epsilon}$ with $\|g(q)-x\|<\epsilon$, the points $g(p)$ and $x$ are $\ll_{\epsilon}$-comparable.

Proof. Suppose that there is a $q \in \mathscr{P}$ such that for every history $h \prec q$ there is a $p \succ h$ and $x \in F_{\epsilon}$ with $\|g(q)-x\|<\epsilon$ such that the points $g(p)$ and $x$ are not $\ll_{\epsilon}$-comparable. Then there is a sequence $p_{t} \in \mathscr{P}$ and $x_{t} \in F_{\epsilon}$ such that $p_{t} \rightarrow q,\left\|g(q)-x_{t}\right\|<\epsilon$ but the points $g\left(p_{t}\right)$ and $x_{t}$ are not $\ll_{\epsilon}$-comparable. As $F_{\epsilon}$ is a finite set we can take $x_{t}$ to be a constant sequence, $x_{t}=x$. Since $g$ has bounded range, by taking a subsequence we may assume that $g\left(p_{t}\right)$ converges, say to a point $y \in G_{g}(q)$. As $g$ has strong CPL, $y$ and $g(q)$ are $\leq-$ comparable. Because $\|g(q)-x\|<\epsilon$, the points $y$ and $x$ are $\ll_{\epsilon}-$ comparable. However, then $g\left(p_{t}\right)$ and $x$ are $\ll_{\epsilon}-$ comparable for $t$ large enough, thus yielding a contradiction.

The following is a straightforward characterization of strong CPL for functions with finite range.

Lemma 6.5. Let $\bar{g}: \mathscr{P} \rightarrow \mathbb{R}^{n}$ be a function with finite range. Then $\bar{g}$ has strong CPL if and only if there is $\bar{\ell}: \mathscr{P} \rightarrow H$ such that (1) for every $p \in \mathscr{P}, \bar{\ell}(p) \prec p$ and (2) for every $p, q \in \mathscr{P}$ if the histories $\bar{\ell}(p)$ and $\bar{\ell}(q)$ are $\preceq$-comparable, the vectors $\bar{g}(p)$ and $\bar{g}(q)$ are $\leq$-comparable.

Proof. Suppose $\bar{g}$ has strong CPL. As in $\S 5$, for every $h \in H$ define $U(h)=\{\bar{g}(p) \mid h \prec p\}$ and for each $p \in \mathscr{P}$ let $h(p)$ be least prefix of $p$ such that $U(h(p))=G_{\bar{g}}(p)$. We show that $h: \mathscr{P} \rightarrow H$ satisfies conditions (1) and (2) of the lemma. Condition (1) is clearly satisfied. To prove (2) take $p, q \in \mathscr{P}$ such that the histories $h(p)$ and $h(q)$ are $\preceq$-comparable. Without loss of generality assume that $h(p) \preceq h(q)$. Then $\bar{g}(q) \in U(h(q)) \subseteq$ $U(h(p))=G_{\bar{g}}(p)$. By strong CPL, $\bar{g}(q)$ is $\leq-$ comparable to $\bar{g}(p)$.

Conversely, suppose that there is a function $\bar{\ell}$ that meets conditions (1) and (2). Take $p \in \mathscr{P}$ and $v \in G_{\bar{g}}(p)$. Then there exists a $q \in \mathscr{P}$ such that $v=\bar{g}(q)$ and $\bar{\ell}(p) \prec q$. As $\bar{\ell}(q) \prec q$ by (1), the histories $\bar{\ell}(p)$ and $\bar{\ell}(q)$ are $\preceq$-comparable. Hence by (2) the vectors $\bar{g}(q)=v$ and $\bar{g}(p)$ are $\leq$-comparable. Thus $\bar{g}$ has strong CPL.

We are now in a position to prove Theorem 6.1. The main instrument we use is induction and recursion on a well-founded relation (see e.g., Jech [9], Theorems 6.10 and 6.11). We construct a well-founded relation on the set of plays that orders $p$ below $q$ if the history $\ell(p)$ is a prefix of the history $\ell(q)$, where the function $\ell$ is as in Lemma 6.4. The discretized payoff function $\bar{g}$ is then defined by recursion on this well-founded relation. Essentially this means that if $p$ is ordered below $q$ then the discretized payoff $\bar{g}(p)$ is defined before $\bar{g}(q)$. By ordering the plays in this way we ensure that whenever $\ell(p)$ is a prefix of $\ell(q)$, the discretized payoff $\bar{g}(p)$ is comparable to $\bar{g}(q)$. This implies that the function $\ell$ satisfies the conditions of Lemma 6.5 , thus $\bar{g}$ has strong CPL.

Define the function $f:[-M, M]^{n} \times 2^{F_{\epsilon}} \rightarrow F_{\epsilon}$ as follows: Let $y \in[-M, M]^{n}$ and $C \subseteq F_{\epsilon}$. If $C$ is a $\leq-$ chain and for each $x \in C$ the points $x$ and $y$ are $\ll_{\epsilon}$-comparable, then let $f(y, C)$ be an element $\bar{y} \in F_{\epsilon}$ satisfying the condition of Lemma 6.3. Otherwise let $f(y, C)$ be any element $v \in F_{\epsilon}$ such that $\|v-y\|<\epsilon$.

Proof of Theorem 6.1. We let $\ell(q)$ be the history as in Lemma 6.4. We construct an $\epsilon$-discretization $\bar{g}$ of the function $g$ with the following property: $\bar{g}(p)$ and $\bar{g}(q)$ are $\leq-$ comparable whenever $\ell(p)$ and $\ell(q)$ are $\preceq$-comparable. By lemma 6.5 this implies that the function $\bar{g}$ has strong CPL.

Let $\triangleleft_{*}$ be any strict well-ordering of $\mathscr{P}$. We define a relation $\triangleleft$ on $\mathscr{P}$ as follows: $p \triangleleft q$ precisely when $[\ell(p) \prec \ell(q)]$ or $\left[\ell(p)=\ell(q)\right.$ and $\left.p \triangleleft_{*} q\right]$. The relation $\triangleleft$ is well-founded.

Now define the function $\bar{g}: \mathscr{P} \rightarrow F_{\epsilon}$ recursively by

$$
\bar{g}(p)=f(g(p),\{\bar{g}(q) \mid q \triangleleft p\}) .
$$


We prove that for each $p, q \in \mathscr{P}, \bar{g}(p)$ and $\bar{g}(q)$ are $\leq-$ comparable whenever the plays $p$ and $q$ are $\triangleleft-$ comparable. Take $p \in \mathscr{P}$ and let $\varphi(p)$ be the statement that for every $q \triangleleft p$, the vectors $\bar{g}(q)$ and $\bar{g}(p)$ are $\leq-$ comparable. Note that if $p$ is the $\triangleleft-$ minimal element of $\mathscr{P}$ then $\varphi(p)$ is trivially true. We prove $\varphi(p)$ by induction on $\triangleleft$. Thus suppose $\varphi(q)$ holds for all $q \triangleleft p$. We must show that $\varphi(p)$ holds.

First we argue that $\{\bar{g}(q) \mid q \triangleleft p\}$ is a $\leq-$ chain. Take $q \triangleleft p$ and $r \triangleleft p$. We must show that $\bar{g}(q)$ and $\bar{g}(r)$ are $\leq-$ comparable. If $q=r$ there is nothing to prove, so assume $q \neq r$. We know that $\ell(q) \preceq \ell(p)$ and $\ell(r) \preceq \ell(p)$, so the histories $\ell(q)$ and $\ell(r)$ are $\preceq$-comparable. Yet this implies that $q$ and $r$ are $\triangleleft$-comparable, say $q \triangleleft r$. Since $\varphi(r)$ holds, however, we conclude that $\bar{g}(q)$ and $\bar{g}(r)$ are $\leq-$ comparable, as desired.

Now we argue that for each $q \triangleleft p$ the points $\bar{g}(q)$ and $g(p)$ are $\ll_{\epsilon}$-comparable. Indeed we have $\ell(q) \preceq$ $\ell(p) \prec p$ and $\|g(q)-\bar{g}(q)\|<\epsilon$. Hence taking $x=\bar{g}(q)$, Lemma 6.4 implies that $g(p)$ and $\bar{g}(q)$ are $\ll_{\epsilon}{ }^{-}$ comparable, as desired.

Therefore, the definition of $f$ implies that, for each $q \triangleleft p$, the points $\bar{g}(q)$ and $\bar{g}(p)$ are $\leq-$ comparable. This proves $\varphi(p)$.

It is now easy to see that, for each $p, q \in \mathscr{P}$, if the histories $\ell(p)$ and $\ell(q)$ are $\preceq$-comparable, then the vectors $\bar{g}(p)$ and $\bar{g}(q)$ are $\leq-$ comparable. Indeed, $\ell(p)$ and $\ell(q)$ are $\preceq$-comparable precisely when $p=q$ or when $p$ and $q$ are $\triangleleft$-comparable.

We now turn to the proof of Theorem 6.2. Recall that $u$ is a bounded payoff function with CPL. The following lemma states that $u$ has a splitting function that exhibits strong CPL. It is constructed by taking the infimum over all splitting functions for $u$ coordinatewise.

LEMma 6.6. The payoff function $u$ has a splitting function s that exhibits strong CPL.

Proof. Given $p \in \mathscr{P}$ define the set

$$
S(p)=\left\{y \in \mathbb{R}^{n} \mid u(p) \leq y \text { and for every } v \in G_{u}(p), v \leq y \text { or } y \leq v\right\} .
$$

Fix some splitting function $s^{\prime}$ for $u$. Then $s^{\prime}(p) \in S(p)$, so $S(p)$ is not empty. For each player $i$ let

$$
s_{i}(p)=\inf _{y \in S(p)} y_{i}
$$

and let $s(p)=\left(s_{1}(p), \ldots, s_{n}(p)\right)$. We prove that $s$ satisfies the requirements of the lemma.

We first argue that $s$ is a splitting function for $u$. We check the three conditions of Definition 3.3: (1) That $u(p) \leq s(p)$ is obvious; (2) There exists a $v \in G_{u}(p)$ such that $s^{\prime}(p) \leq v$, hence $s(p) \leq v$; (3) Take any $v \in G_{u}(p)$. Two cases are possible: (A) For every $y \in S(p)$ it holds that $v \leq y$. In this case $v \leq s(p)$. (B) There exists a $y \in S(p)$ such that $y \leq v$. In this case $s(p) \leq v$. Hence we have $v \leq s(p)$ or $s(p) \leq v$, as desired.

We show that $s$ has strong CPL. Take $p \in \mathscr{P}$ and $y \in G_{s}(p)$. Suppose that the sequence $\left(p_{k}, s\left(p_{k}\right)\right)$ converges to $(p, y)$. Below we show that, for every $\epsilon>0$, there exists a $K_{\epsilon} \in \mathbb{N}$ such that for each $k \geq K_{\epsilon}$ the points $s\left(p_{k}\right)$ and $s(p)$ are $\leq_{\epsilon}$-comparable. This is sufficient, because then there exist infinitely many $k \in \mathbb{N}$ with $s\left(p_{k}\right) \leq_{\epsilon} s(p)$ or infinitely many $k \in \mathbb{N}$ with $s(p) \leq_{\epsilon} s\left(p_{k}\right)$. In the first case $y \leq_{\epsilon} s(p)$, and in the second case $s(p) \leq_{\epsilon} y$. Since this is true for every $\epsilon>0$, we conclude that $y \leq s(p)$ or $s(p) \leq y$.

Fix an $\epsilon>0$. It can be seen that there exists a $K_{\epsilon} \in \mathbb{N}$ such that for $k \geq K_{\epsilon}$ the set $G_{u}\left(p_{k}\right)$ is contained in the $\epsilon$-neighborhood of $G_{u}(p)$. That is, for each $v \in G_{u}\left(p_{k}\right)$ there exists $v^{\prime} \in G_{u}(p)$ such that $\left\|v^{\prime}-v\right\|<\epsilon{ }^{4}$

Fix a $k \geq K_{\epsilon}$. Each $v \in G_{u}\left(p_{k}\right)$ is $\leq-$ comparable to $s\left(p_{k}\right)$ since $s$ is a splitting function for $u$. Moreover, $v$ is also $\leq_{\epsilon}$-comparable to $s(p)$ since $v$ is in the $\epsilon$-neighborhood of $G_{u}(p)$.

Suppose $\neg\left(s(p) \leq_{\epsilon} s\left(p_{k}\right)\right)$. We argue that $s\left(p_{k}\right) \leq_{\epsilon} s(p)$. Let $z=\left(z_{1}, \ldots, z_{n}\right)$ where $z_{i}=\min \left\{s_{i}(p)+\epsilon\right.$, $\left.s_{i}\left(p_{k}\right)\right\}$. We show that $z \in S\left(p_{k}\right)$.

First we argue that $u\left(p_{k}\right) \leq z$. We know that $u\left(p_{k}\right) \leq s\left(p_{k}\right)$. Our supposition implies that $\neg\left(s(p) \leq_{\epsilon} u\left(p_{k}\right)\right)$. Yet because $s(p)$ is $\leq_{\epsilon}$-comparable to $u\left(p_{k}\right) \in G_{u}\left(p_{k}\right)$, we must have $u\left(p_{k}\right) \leq_{\epsilon} s(p)$. Now we conclude that $u\left(p_{k}\right) \leq z$.

Take a $v \in G_{u}\left(p_{k}\right)$. We argue $v$ and $z$ are $\leq-$ comparable. If $s\left(p_{k}\right) \leq v$ then $z \leq v$. If $v \leq s\left(p_{k}\right)$ then our supposition implies that $\neg\left(s(p) \leq_{\epsilon} v\right)$. Since $v$ is $\leq_{\epsilon}$-comparable to $s(p)$, however, we must have $v \leq_{\epsilon} s(p)$. Thus $v \leq z$.

This proves that $z \in S\left(p_{k}\right)$. However, then $s\left(p_{k}\right) \leq z \leq_{\epsilon} s(p)$.

To conclude, $s\left(p_{k}\right)$ is $\leq_{\epsilon}$-comparable to $s(p)$, as desired.

\footnotetext{
${ }^{4}$ Indeed, suppose otherwise. By passing to a subsequence, if necessary, we obtain a sequence of $v_{k} \in G_{u}\left(p_{k}\right)$ such that for all $k \in \mathbb{N}$ and all $v^{\prime} \in G_{u}(p)$ it holds that $\left\|v^{\prime}-v_{k}\right\| \geq \epsilon$. The sequence $v_{k}$ is bounded since the payoffs are bounded. Hence, it has a convergent subsequence. Replacing the sequence by a subsequence we can assume that $v_{k}$ converges, say to a point $v \in \mathbb{R}^{n}$. Since $G_{u}$ is a closed set, we have $v \in G_{u}(p)$. Hence $\left\|v-v_{k}\right\| \geq \epsilon$ for all $k \in \mathbb{N}$, leading to a contradiction.
} 
By the above lemma $u$ has a splitting function that exhibits strong CPL. Fix one such splitting function $s$. Clearly, $-M \leq s_{i}(p) \leq M$ for each $p$ and each $i$. The next approximation result is the counterpart of Lemma 6.4 for the CPL rather than strong CPL.

Lemma 6.7. For each $q \in \mathscr{P}$, there exists a history $\ell^{*}(q) \prec q$ such that, for each $p \succ \ell^{*}(q)$ and each $x \in F_{\epsilon}$ with $\|s(q)-x\|<\epsilon$, the points $u(p)$ and $x$ are $\ll_{\epsilon}$-comparable.

Proof. Suppose that there is a $q \in \mathscr{P}$ such that for every history $h \prec q$ there is a $p \succ h$ and $x \in F_{\epsilon}$ with $\|s(q)-x\|<\epsilon$ such that the points $u(p)$ and $x$ are not $\ll_{\epsilon}$-comparable. Then there is a sequence $p_{t} \in \mathscr{P}$ and $x_{t} \in F_{\epsilon}$ such that $p_{t} \rightarrow q,\left\|s(q)-x_{t}\right\|<\epsilon$ but the points $u\left(p_{t}\right)$ and $x_{t}$ are not $\ll_{\epsilon}$-comparable. As $F_{\epsilon}$ is a finite set we can take $x_{t}$ to be a constant sequence, $x_{t}=x$. Since $u$ has bounded range, by taking a subsequence we may assume that $u\left(p_{t}\right)$ converges, say to a point $y \in G_{u}(q)$. Because $u$ has CPL, $y$ are $s(q)$ are $\leq-$ comparable. As $\|s(q)-x\|<\epsilon$, the points $y$ and $x$ are $\ll_{\epsilon}$-comparable. But then $u\left(p_{t}\right)$ and $x$ are $\ll_{\epsilon}$-comparable for $t$ sufficiently large, thus yielding a contradiction.

We are now in a position to prove Theorem 6.2. The proof uses the function $f$ introduced earlier, and the fact that the splitting function $s$ has strong CPL, and hence admits an $\epsilon$-discretization by Theorem 6.1 .

Proof of Theorem 6.2. By Theorem 6.1 the splitting function $s$ has an $\epsilon$-discretization, i.e., a strong CPL function $\bar{s}: \mathscr{P} \rightarrow F_{\epsilon}$ such that $\|s(p)-\bar{s}(p)\|<\epsilon$. Take $\bar{\ell}(p)$ to be a history as in Lemma 6.5 for $\bar{g}=\bar{s}$. Let $\ell^{*}(p)$ be as in Lemma 6.7. Now let $m(p)$ denote the longer of the histories $\bar{\ell}(p)$ and $\ell^{*}(p)$.

For $p \in \mathscr{P}$ define

$$
C(p)=\{\bar{s}(q) \mid q \in \mathscr{P}, m(q) \prec p\} .
$$

Note that the set $C(p)$ is a $\leq-$ chain by the choice of the function $m$. Moreover, $u(p)$ is $\ll_{\epsilon}$-comparable to every point in $C(p)$ by Lemma 6.7 .

Let $\mathscr{P}_{0}=\left\{p \in \mathscr{P} \mid \bar{s}(p) \ll_{\epsilon} u(p)\right\}$ and let $\mathscr{P}_{1}$ be the complement of $\mathscr{P}_{0}$ in $\mathscr{P}$. Define

$$
\bar{u}(p)= \begin{cases}\bar{s}(p) & \text { if } p \in \mathscr{P}_{0} \\ f(u(p), C(p)) & \text { if } p \in \mathscr{P}_{1} .\end{cases}
$$

Clearly, $\bar{u}$ has finite range. Now we argue that $\|\bar{u}(p)-u(p)\|<\epsilon$. For $p \in \mathscr{P}_{1}$ this follows from the definition of $f$. Now take $p \in \mathscr{P}_{0}$. Then for every $i$ we have $\bar{s}_{i}(p)-\epsilon<u_{i}(p) \leq s_{i}(p)<\bar{s}_{i}(p)+\epsilon$. Hence $\left|u_{i}(p)-\bar{s}_{i}(p)\right|<\epsilon$. Therefore $\left|u_{i}(p)-\bar{u}_{i}(p)\right|<\epsilon$. This shows that $\bar{u}$ is an $\epsilon$-discretization of $u$.

We show that $\bar{u}$ has CPL with $\bar{s}$ being the splitting function. We verify the three conditions of Definition 3.3:

(1) For $p \in \mathscr{P}_{0}$ we have $\bar{u}(p)=\bar{s}(p)$. For $p \in \mathscr{P}_{1}$ it is not the case that $\bar{s}(p) \ll_{\epsilon} u(p)$. It follows by the definition of $f$ and because $\bar{s}(p) \in C(p)$ that $\bar{u}(p) \leq \bar{s}(p)$.

(2) For $p \in \mathscr{P}_{0}$ we have $\bar{u}(p)=\bar{s}(p)$. The condition is satisfied since $\bar{u}(p) \in G_{\bar{u}}(p)$. Take a $p \in \mathscr{P}_{1}$. There exists a $v \in G_{u}(p)$ such that $s(p) \leq v$. Then $\bar{s}(p) \ll_{\epsilon} v$. Let $\left\{\left(p_{k}, u\left(p_{k}\right)\right)\right\}_{k \in \mathbb{N}}$ be a sequence converging to $(p, v)$. Then $m(p) \prec p_{k}$ and $\bar{s}(p) \ll_{\epsilon} u\left(p_{k}\right)$ for large $k$. Hence, by the definition of $f$ we have $\bar{s}(p) \leq \bar{u}\left(p_{k}\right)$ for large $k$. Replacing the sequence by a subsequence, if necessary, assume that $\bar{u}\left(p_{k}\right)=\bar{v}$ for every $k$. Then $\bar{s}(p) \leq \bar{v}$, and $\bar{v} \in G_{\bar{u}}(p)$.

(3) Take a $(p, \bar{v}) \in G_{\bar{u}}$ and let the sequence $\left\{\left(p_{k}, \bar{u}\left(p_{k}\right)\right)\right\}_{k \in \mathbb{N}}$ converge to $(p, \bar{v})$. Suppose first that there exists a subsequence of $p_{k}$ in $\mathscr{P}_{0}$. Replace the sequence with the subsequence. Since $\bar{u}\left(p_{k}\right)=\bar{s}\left(p_{k}\right)$ for each $k$ and since $\bar{s}$ has strong CPL, $\bar{v}$ is $\leq-$ comparable to $\bar{s}(p)$. Now suppose that only finitely many elements of $p_{k}$ are in $\mathscr{P}_{0}$. Then $p_{k} \in \mathscr{P}_{1}$ for $k$ large. Moreover $m(p) \prec p_{k}$ and hence $\bar{s}(p) \in C\left(p_{k}\right)$ for large $k$. By the definition of $f$, the vectors $\bar{u}\left(p_{k}\right)$ and $\bar{s}(p)$ are $\leq-$ comparable. Hence $\bar{v}$ and $\bar{s}(p)$ are $\leq-$ comparable.

7. Concluding remarks. In this section we discuss two straightforward extensions of Theorem 4.2 for a payoff function with finite range. For the sake of simplicity we further assume throughout this section that the payoff function has no ties (for the definition of a tie see §5).

7.1. Induced plays and upper semicontinuity plays. The construction of SPE strategies in $\S 5$ allows us to improve on Theorem 4.2 as follows.

Corollary 7.1. Let $u=\left(u_{1}, \ldots, u_{n}\right)$ be a payoff function with finite range, no ties, and common preferences at the limit. Let the strategy profile $\sigma$ be as in Lemma 5.2. Then $\sigma$ is an SPE with the following additional property: For every $h \in H$ and for every player $i$, the payoff function $u_{i}$ is upper semicontinuous at the induced play $\pi(\sigma, h)$. 
The corollary can be proven as follows: We can assume without loss of generality that each $u_{i}$ only takes integer values. Let $s^{\prime}$ be any splitting function for $u$. Define a new splitting function $s$ for $u$ as follows. Take a play $p$ and a player $i$. If $u_{i}(p)=s_{i}^{\prime}(p)$ and there is a $v \in G_{u}(p)$ with $v_{i}>u_{i}(p)$, then let $s_{i}(p)=s_{i}^{\prime}(p)+0.5$. Otherwise, let $s_{i}(p)=s_{i}^{\prime}(p)$. Since $u$ has no ties, $s$ is indeed a splitting function for $u$.

By definition, the function $s$ has the following additional property: For each player $i$ and each $p \in \mathscr{P}$, if $u_{i}(p)=s_{i}(p)$, then $u_{i}$ is upper semicontinuous at $p$.

Let $\sigma$ be a strategy profile as in Lemma 5.2. As shown in the proof of Theorem 4.2, the strategy profile $\sigma$ is an SPE. Take a history $h \in H$ and let $p=\pi(\sigma, h)$. Let $h^{\prime}$ be the longer of the two histories $h$ and $h(p)$, where $h(p)$ is defined as in $\S 5$. Now Lemma 5.5 implies that $u\left(\pi\left(\sigma, h^{\prime}\right)\right) \geq s(p)$. By the choice of $h^{\prime}$, we have $\pi\left(\sigma, h^{\prime}\right)=p$. Thus $u(p) \geq s(p)$, and hence $u(p)=s(p)$. The choice of the splitting function $s$ now implies that each $u_{i}$ is upper semicontinuous at $p$. This completes the proof of the corollary.

One implication of the corollary is that for CPL payoff functions with finite range and no ties, the set of upper semicontinuity plays is dense as a subset of $\mathscr{P}$. We remark that the denseness of upper semicontinuity plays alone is not sufficient for the existence of an SPE, not even of an $\epsilon-\mathrm{SPE}$ in behavioral strategies. For a counterexample, see Flesch and Predtetchinski [6].

Corollary 7.1 cannot be extended to the payoff functions with infinite range, since then the set of upper semicontinuity plays might be empty even in a one player game. To see this consider a one player game with the action set $A=\{1,2\}$. Let $E$ be the set of eventually constant plays. For $p$ in $E$ let $e(p)$ be the period at which $p$ becomes constant. Define $u(p)$ to be 0 if $p \notin E$, and $2-1 /(e(p)+1)$ if $p \in E$. Then $u$ is nowhere upper semicontinuous. To see this, take any $p=\left(a_{0}, a_{1}, \ldots\right)$. For any $t \in \mathbb{N}$ let $p_{t}=\left(a_{0}, \ldots, a_{t-1}, b_{t}, b_{t}, b_{t}, \ldots\right)$ where $b_{t} \neq a_{t-1}$. Then $e\left(p_{t}\right)=t$. Hence $\lim \sup _{t \rightarrow \infty} u\left(p_{t}\right)=2>u(p)$.

7.2. Splitting functions. For a function $u: \mathscr{P} \rightarrow \mathbb{R}^{n}$ let $\Omega(u)$ denote the game with the payoff function $u=\left(u_{1}, \ldots, u_{n}\right)$.

CoRollary 7.2. Let $u=\left(u_{1}, \ldots, u_{n}\right)$ be a payoff function with finite range, no ties, and common preferences at the limit. Let $s$ be an associated splitting function. Let the strategy profile $\sigma$ be as in Lemma 5.2. Then $\sigma$ is an SPE in both games $\Omega(u)$ and $\Omega(s)$.

The proof of the corollary is as follows: Let $\sigma$ be a strategy profile as in Lemma 5.2. As shown in the proof of Theorem 4.2, the strategy profile $\sigma$ is an SPE for the game $\Omega(u)$. For every history $h \in H$, the payoff function $u$ is upper semicontinuous at the induced play $\pi(\sigma, h)$, by Corollary 7.1. Hence, for every $h \in H$, we have $u(\pi(\sigma, h))=s(\pi(\sigma, h))$.

Now consider a strategy $\eta_{i}$ for some player $i$ and a history $h \in H$. Let $p=\pi\left(\sigma / \eta_{i}, h\right)$. Let $h^{\prime}$ be the longer of the two histories $h$ and $h(p)$. Then by Lemma 5.5 we have $u\left(\pi\left(\sigma, h^{\prime}\right)\right) \geq s(p)$. Since $\sigma$ is an SPE in $\Omega(u)$ and since $h^{\prime}$ is reached from $h$ by a unilateral deviation to $\eta_{i}$, we have $u_{i}(\pi(\sigma, h)) \geq u_{i}\left(\pi\left(\sigma, h^{\prime}\right)\right)$. Combining the two inequalities we obtain

$$
s_{i}(\pi(\sigma, h))=u_{i}(\pi(\sigma, h)) \geq u_{i}\left(\pi\left(\sigma, h^{\prime}\right)\right) \geq s_{i}(p)=s_{i}\left(\pi\left(\sigma / \eta_{i}, h\right)\right) .
$$

This completes the proof of the corollary.

Note that the sets of SPEs in $\Omega(u)$ and $\Omega(s)$ are not nested, i.e., neither one is generally a superset of the other. This is illustrated by the following example.

EXAMPLE 7.3. Consider a game with only one player and the action set $A=\{1,2\}$. Let $E^{2}$ denote the set of plays in which, after some period, only action 2 is played. The payoff function is given by $u(p)=1$ if $p \in E^{2}$, and $u(p)=0$ otherwise. Observe that $u$ satisfies CPL with the splitting function $s(p)=1$ for all $p \in \mathscr{P}$. In particular, condition (2) of Definition 3.3 is satisfied because the set $E^{2}$ is dense in $\mathscr{P}$. Obviously, any strategy is an SPE in $\Omega(s)$, but not in $\Omega(u)$.

Now consider a game with two players playing alternatingly and with the action set $A=\{1,2\}$. Let $E^{a}$ denote the set of plays in which, after some period, only action $a$ is played. The payoff function is given by $u(p)=(2,2)$ if $p \in E^{2}$ and $u(p)=(1,1)$ if $p \in E^{1}$ and $u(p)=(0,0)$ otherwise. Observe that $u$ satisfies CPL with the splitting function $s$ given by $s(p)=(2,2)$ if $p \in E^{2}$ and $s(p)=(1,1)$ if $p \in E^{1}$ and $s(p)=(1.5,1.5)$ otherwise. The strategy profile in which both players always choose action 1 is an SPE in $\Omega(u)$, but not in $\Omega(s)$, because in $\Omega(s)$ each player can guarantee a payoff of at least 1.5 (by choosing actions 1 and 2 alternatingly). $\triangleleft$

Interestingly, Corollary 7.2 establishes the existence of an SPE for a class of payoff functions that is larger than just CPL payoff functions. Indeed, there exist games where the payoff function exhibits CPL, whereas a 
corresponding splitting function does not, as the example below illustrates. This calls for a precise characterization of all payoff functions that are a splitting function for some CPL payoff function. We leave this question for further research.

EXAMPLE 7.4. We construct payoff functions $u$ and $s$ with the following properties: $u$ exhibits CPL, $s$ is a splitting function associated with $u$, and $s$ does not exhibit CPL.

There are two players. At any history, player 1 is the active player. The set of actions is $\{a, b, c\}$. Let $B$ denote the set of plays in which player 1 chooses action $b$ at least once, and action $c$ is not chosen before the first occurrence of $b$. Similarly, let $C$ denote the set of plays in which player 1 chooses action $c$ at least once, and action $b$ is not chosen before the first occurrence of $c$. Furthermore, let $B^{\infty}$ denote the set of plays in which player 1 chooses action $b$ infinitely often, and let $C^{\infty}$ denote the set of plays in which player 1 chooses action $c$ infinitely often.

The payoffs are given for each play $p$ as follows:

$$
u(p)= \begin{cases}(0,0) & \text { if } p=(a, a, a, \ldots) \\ (0,0) & \text { if } p \in B \text { and } p \notin B^{\infty} \\ (2,1) & \text { if } p \in B \text { and } p \in B^{\infty} \\ (0,0) & \text { if } p \in C \text { and } p \notin C^{\infty} \\ (1,2) & \text { if } p \in C \text { and } p \in C^{\infty}\end{cases}
$$

Note that $G_{u}(p)=\{(0,0),(2,1),(1,2)\}$ for $p=(a, a, a, \ldots)$, and $G_{u}(p)=\{(0,0),(2,1)\}$ for $p \in B$, and $G_{u}(p)=\{(0,0),(1,2)\}$ for $p \in C$. It is straightforward to verify that $u$ exhibits CPL with the splitting function $s$ given by:

$$
s(p)= \begin{cases}(1,1) & \text { if } p=(a, a, a, \ldots) \\ (2,0) & \text { if } p \in B \text { and } p \notin B^{\infty} \\ (2,1) & \text { if } p \in B \text { and } p \in B^{\infty} \\ (0,2) & \text { if } p \in C \text { and } p \notin C^{\infty} \\ (1,2) & \text { if } p \in C \text { and } p \in C^{\infty}\end{cases}
$$

Clearly, $s$ does not exhibit CPL, as $G_{s}(p)=\{(1,1),(2,0),(2,1),(0,2),(1,2)\}$ for $p=(a, a, a, \ldots) . \triangleleft$

Acknowledgments. The authors thank the participants at the game theory seminar in Paris, the MAGTA 2014 conference, the 5th York Symposium on Game Theory, and the economics seminar in York, as well as the associate editor, and two referees for their useful comments.

\section{References}

[1] Alós-Ferrer C, Ritzberger K (2016) Characterizing existence of equilibrium for large extensive form games. J. Math. Econom., 62:5-12.

[2] Flesch J, Kuipers J, Mashiah-Yaakovi A, Schoenmakers G, Solan E, Vrieze K (2010) Perfect-information games with lower semicontinuous payoffs. Math. Oper. Res. 35(4):742-755.

[3] Flesch J, Kuipers J, Schoenmakers G, Vrieze K (2013) Subgame-perfection in free transition games. Eur. J. Oper. Res. 228:201-207.

[4] Flesch J, Kuipers J, Mashiah-Yaakovi A, Shmaya E, Schoenmakers G, Solan E, Vrieze K (2014) Non-existence of subgame-perfect $\epsilon$-equilibrium in perfect information games with infinite horizon. Int. J. Game Theory 43:945-951.

[5] Flesch J, Predtetchinski A (2015) On refinements of subgame perfect $\epsilon$-equilibrium. Int. J. Game Theory. Forthcoming.

[6] Flesch J, Predtetchinski A (2016) Subgame-perfect $\epsilon$-equilibria in perfect information games with sigma-discrete discontinuities. J. Econom. Theory 61(3):479-495.

[7] Fudenberg D, Levine D (1983) Subgame-perfect equilibria of finite and infinite-horizon games. J. Econom. Theory 31:251-268

[8] Harris C (1985) Existence and characterization of perfect equilibrium in games of perfect information. Econometrica 53:613-628.

[9] Jech T (2006) Set Theory, Third Edition (Springer, Berlin).

[10] Laraki R, Maitra AP, Sudderth WD (2013) Two-person zero-sum stochastic games with semicontinuous payoff. Dynamic Games Appl. 3:162-171.

[11] Mashiah-Yaakovi A (2014) Subgame perfect equilibria in stopping games. Int. J. Game Theory 43:89-135.

[12] Maitra AP, Sudderth WD (2007) Subgame-perfect equilibria for stochastic games. Math. Oper. Res. 32(3):711-722.

[13] Martin DA (1975) Borel determinacy. Ann. Math. 2:363-370.

[14] Mertens J-F (1987) Repeated games. Proc. Int. Congress of Mathematicians, (Berkeley, California, 986), 1528-1577 (American Mathematical Society, Providence, RI).

[15] Purves RA, Sudderth WD (2011) Perfect information games with upper semicontinuous payoffs. Math. Oper. Res. 36(3):468-473.

[16] Solan E, Vieille N (2003) Deterministic multi-player Dynkin games. J. Math. Econom. 39:911-929.

[17] Solan E (2005) Subgame-perfection in quitting games with perfect information. Math. Oper. Res. 30(1):51-72. 